\title{
Gene conversion and purifying selection of a placenta-specific ERV-V envelope gene during simian evolution Anders L Kjeldbjerg ${ }^{\dagger 1}$, Palle Villesen ${ }^{\dagger 2}$, Lars Aagaard ${ }^{1}$ and Finn Skou Pedersen*1
}

\author{
Address: ${ }^{1}$ Department of Molecular Biology, University of Aarhus, DK-8000 Aarhus C, Denmark and 2Bioinformatics Research Center, University \\ of Aarhus, DK-8000 Aarhus C, Denmark \\ Email: Anders L Kjeldbjerg - alk@mb.au.dk; Palle Villesen - palle@birc.au.dk; Lars Aagaard - laa@mb.au.dk; \\ Finn Skou Pedersen* - fsp@mb.au.dk \\ * Corresponding author †Equal contributors
}

Published: 30 September 2008

BMC Evolutionary Biology 2008, 8:266 doi:10.1 186/147/-2/48-8-266
Received: II April 2008

Accepted: 30 September 2008

This article is available from: http://www.biomedcentral.com//47/-2/48/8/266

(c) 2008 Kjeldbjerg et al; licensee BioMed Central Ltd.

This is an Open Access article distributed under the terms of the Creative Commons Attribution License (http://creativecommons.org/licenses/by/2.0), which permits unrestricted use, distribution, and reproduction in any medium, provided the original work is properly cited.

\begin{abstract}
Background: Most human endogenous retroviruses (HERVs) invaded our genome at least 25 million years ago. The majority of the viral genes are degenerated, since no selection preserves them within the genome. However, a few intact and very old HERV genes exist, and likely are beneficial for the host. We here address evolutionary aspects of two HERV-V envelope genes, $E N V V I$ and ENVV2, located in tandem and containing a long open reading frame.

Results: The ENVV2 gene is preserved with an intact reading frame during simian evolution, but none of the ENVV genes are found in the prosimian species tested. While we observe many transposon insertions in the gag and pol regions of the ERV-V2 provirus, the ENVV2 genes have escaped transposon crossfire in all species tested. Additional analysis of nucleotide substitutions provides further strong evidence of purifying selection on the ENVV2 gene during primate evolution. The other copy, ENVVI, seems to be involved in gene conversion of the major part of the envelope. Furthermore, ENVVI and ENVV2 show placenta-specific expression in human and a baboon species.

Conclusion: Our analyses show that ERV-V entered our genome after the split between simian and prosimian primates. Subsequent purifying selection and gene conversion have preserved two copies of the ENVV envelope gene in most species. This is the first case of gene conversion involving long open reading frames in HERVs. Together with the placenta-specific expression of the human and baboon ENVVI and ENVV2 envelope genes, these data provide strong evidence of a beneficial role for the host.
\end{abstract}

\section{Background}

Upon retrovirus infection of somatic cells, the integrated provirus will not be passed on to the host progeny as a part of the genome. However, following infection of the germ line, the integrated provirus will be transmitted to the offspring. Consequently, progeny developed from infected germ cells will carry the provirus as part of their genome, and it will be transmitted vertically through generations as an endogenous retrovirus (ERV). Each independent germ line infection event defines a novel ERV family, which may increase its copy number due to intracellular retrotransposition [1] or extracellularly via re- 
infection [2], and in the end each ERV infection results in a few to several hundred genomic copies [3]. Most HERVs invaded our genome at least 25 million years ago (mya) $[4,5]$, after separation of Old World and New World monkeys around 43 mya [6]. ERVs that entered the human genome before the split of human (Homo sapiens) and chimpanzee (Pan troglodytes) are characterized as ancient HERVs. However, some HERVs, characterized as modern HERVs, are human-specific and have entered the human genome after the Homo sapiens/Pan troglodytes split. Further, some HERV loci have been reported to show insertional polymorphism in the human genome, even one HERV locus has entered the human genome less than 1 mya [7].

Generally HERV-encoded genes are thought to be inactivated by negative selection, followed by degeneration due to mutational decay during evolution. However, a few HERV loci do still maintain intact open reading frames of viral genes, indicating either recent integration or ongoing purifying selection. No replication-competent HERVs have yet been described, although fully intact members of the HERV-K group have been reported [7]. Nevertheless, trans-complementation and recombination of human HERV-K loci can generate functional HERV-K elements, indicating that human cells still have the potential to produce infectious retrovirus particles $[8,9]$. However, other mammalian species such as mouse, cat and pig harbor many modern ERVs which are still replication-competent [4].

Intact envelope genes have been shown to be transcribed in several healthy tissues [10], and the conservation in an otherwise degenerated HERV locus has led to speculations about a likely beneficial role for the host. These include (i) protecting the fetus due to immunomodulatory properties via an immunosuppressive domain located in the TM subunit of the envelope $[11,12]$, (ii) preventing presentday retroviral infections by inhibiting cell entry of related exogenous retroviruses that use a common surface recep- tor, a process called receptor interference in which the receptor-binding-domain of SU blocks the receptor $[13,14]$, or (iii) being used as triggers to provide cell-cell fusion in which the fusion machinery of TM is activated by binding of SU to a cellular receptor. In particular three HERV envelope genes can induce cell-cell fusion in vitro, syncytin 1 [15,16], syncytin 2 [17], and EnvPb1 [18]. All three are candidates for having a beneficial function because they are evolutionarily conserved and have undergone purifying selection during primate evolution $[17,19,20]$. Furthermore all single nucleotide polymorphisms (SNPs) within the three envelope genes are either synonymous or they do not influence fusiogenicity $[20,21]$.

Syncytin 1 and syncytin 2 show placenta-specific expression $[10,15,22,23]$, which may be an implication of a physiological role of HERV envelope proteins in mediating cell-cell fusion in placenta forming the syncytiotrophoblast. In fact inhibition of syncytin 1 in human cytotrophoblasts leads to a decrease in cell fusion [24], indicating a plausible physiological role of syncytin 1 in placenta development. Syncytin 2 might protect the fetus against the mother's immune system due to immunosuppressive properties [11], but so far none of them have been well enough characterized to draw functional conclusions.

During a screen of the human genome for retroviral open reading frames [25], we identified a new group, dubbed HERV-V [18], containing two almost identical envelope genes. HERV-V was recently proposed to be a degenerate syncytin [11]. However, we here demonstrate that selection has preserved at least one of the envelope genes through simian evolution, and that the other envelope gene has been partly preserved by gene conversion.

\section{Results}

The two, almost identical, HERV-V envelope genes are both located on chromosome 19q13.41 with a distance

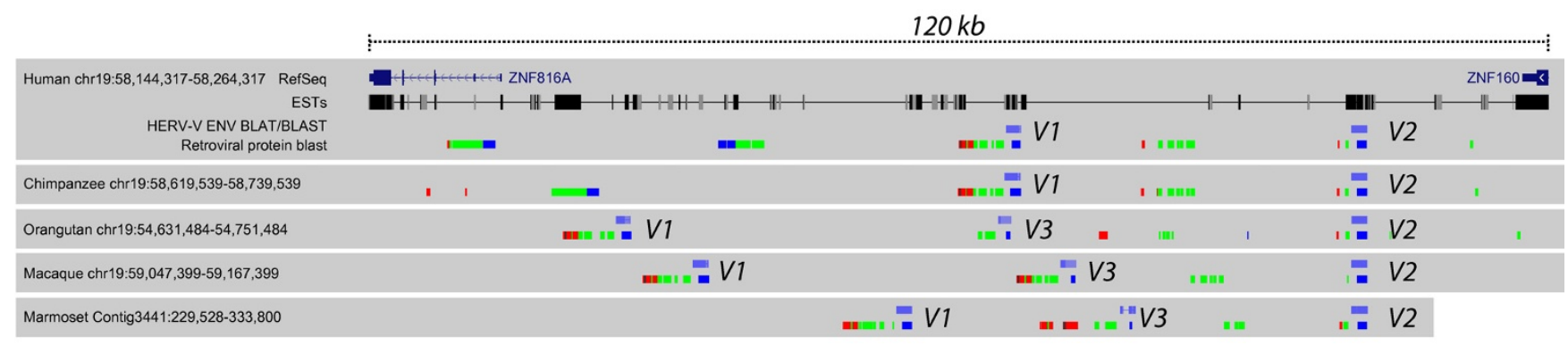

\section{Figure I}

ENVV2 in primate genomes. The location of ERV-V loci on chromosome 19 in five primate species. The ENVV genes are shown in light blue whereas similarity to other HERV proteins are coded as gag (red), pol (green) and env (blue). 
between the two loci of $\sim 34 \mathrm{~kb}$ (Figure 1) [25]. In order to distinguish the two envelopes from each other, we propose naming one locus HERV-V1 and its envelope ENVV1 (chr19: 58209156-58210586, hg18) and the other locus HERV-V2 and its envelope ENVV2 (chr19: 5824431758245921, hg18). ENVV1 has a 477 amino-acid long open reading frame and ENVV2 has an open reading frame containing 535 amino acids. Variation is only observed in the C-terminus of the genes by a 60 aminoacid truncation of $E N V V 1$, due to a one nucleotide insertion leading to a frame shift. A tBLASTn search shows no other closely related sequences in the human genome and a sensitive BLASTn search using the cross-species LTR consensus show no sign of solitary LTRs. Further, both envelopes show high similarity in the C-terminal part to two other HERV envelope genes: ERVWE1 (syncytin 1) and HERV-FRD (syncytin 2) (Env-FRD) [25]. Phylogenetic comparison indicates that HERV-V is a small new family of ERVs, most closely related to that of MER66, MER84 and Z69907 families [18].

\section{The ENVV envelope genes are conserved in simians}

Using tBLASTn and BLAT we found ENVV1 and ENVV2 at homologous positions on chromosome 19 in chimpanzee (Pan troglodytes), Sumatran orangutan (Pongo pygmaeus abelii), rhesus macaque (Macaca mulatta) and marmoset (Callithrix jacchus, Contig3441) (Figure 1). In all species, the ENVV2 gene is intact, whereas the ENVV1 gene is preserved only in chimpanzee and rhesus macaque, where it exhibits a full-length ORF with no C-terminal truncation or stop codons. In orangutan, rhesus macaque and marmoset, ENVV1 and ENVV2 are separated by $45-65 \mathrm{~kb}$ and exhibit a third ERV-V locus in between, named ERV-V3. No ENVV homologues were detected in any genome more distant than those of New World monkeys.

Additionally, we screened a primate DNA panel with PCR primers flanking either the ENVV1 or ENVV2 envelope genes (Figure 2A) and were able to amplify the expected 2.5-kb amplicon from Hominoidea, Old World monkeys and New World monkeys (Borneo orangutan (Pongo pygmaeus), African green monkey (Cercopithecus aethiops)and squirrel monkey (Saimiri sciureus), respectively). In addition, the ENVV3 was also detected in the African green monkey by an internal 500 bp PCR fragment (data not shown). Interestingly, sequencing of the PCR products revealed that the ENVV2 gene is preserved in all species analyzed and ENVV1 is preserved in Old World monkeys (Cercopithecoidea), whereas orangutan and squirrel monkey only show preservation of ENVV2 (Figure 2B).

We also attempted to PCR amplify the $2.5 \mathrm{~kb}$ fragment from the prosimian ring-tailed lemur (Lemur catta). However, no amplicon emerged (Figure 2A), in agreement with a database search on the accessible prosimian genome-sequencing-trace reads. In summary, both database searches and PCR amplification of ENVV1, ENVV2 and ENVV3 indicated an integration of ERV-V after the simian-prosimian (Anthropoidea-Strepsirrhini) split (77 mya[6]) and before the Catarrhini-Platyrrhini split (43 mya [6]), immediately followed by a new infection/reinfection and a duplication of the genome surrounding the ERV-V locus, or vice versa. Similarity between the 5' and 3' genomic regions surrounding the ERV-V1 and ERV-V2 loci suggests that a genomic duplication has assisted in generating ERV-V1 and ERV-V2, e.g. a 200 bp fragment upstream of HERV-V2 (chr19: 58247800-58247999, hg18) has $77 \%$ similarity to a region upstream of HERVV1. The same is also found in chimpanzee and rhesus macaque. This similarity cannot be found in the regions flanking the ERV-V3 locus. However, the ERV-V3 locus is absent in Hominidae, indicating a deletion of the ERV-V3 before the human and chimpanzee separation (Figure 2B).

In theory, LTR sequence divergence can be used as a rough indicator of integration time, since the two LTRs are identical at the time of integration, but the estimate might be problematic because of confounding processes such as recombination and conversion [26,27]. The LTR divergences (human: 0.284, chimp: 0.271 and orangu$\tan : 0.370)$ indicate that the ERV-V locus descended from an old integration by being even more distinct than HERV-FRD (syncytin 2) LTRs (human: 0.225, chimp: 0.227 and macaque: 0.316 ] [28], which integrated on the same branch of the primate phylogeny. However our LTRanalysis indicates unrealistic divergence times for both ERV-V2 (96-217 mya) and syncytin 2 (83-152 mya) showing the difficulties in using LTR divergence for dating old, conserved ERV integrations.

\section{Gene conversion between ERV-V envelope genes}

On the basis of the finding that both ERV-V copies can be dated back to before the Catarrhini-Platyrrhini split, we expect the evolutionary distance between the paralogue ENVV genes (ENVV1-ENVV2) to be larger than the evolutionary distance between orthologue $E N V V$ genes (ENVV1-ENVV1 and ENVV2-ENVV2) within the most distant species (e.g. human/marmoset), resulting in two monophyletic groups, each following the primate phylogeny (an ENVV1 group and an ENVV2 group, Figure 3A). To our surprise, we observed that the paralogue genes were more closely related than the orthologue genes between the most distant species, Hominidae/Cercopithecidae and Platyrrhini (Kimura-2-p. distances: ENVV1ENVV2, $0.024 \pm 0.004$ vs ENVV2-ENVV2 and ENVV1ENVV1, $0.140 \pm 0.006)$.

A clear change in the substitution pattern was observed in the ENVV1/ENVV2 alignment, and a subsequent gene- 
A

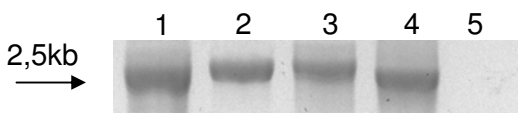

B

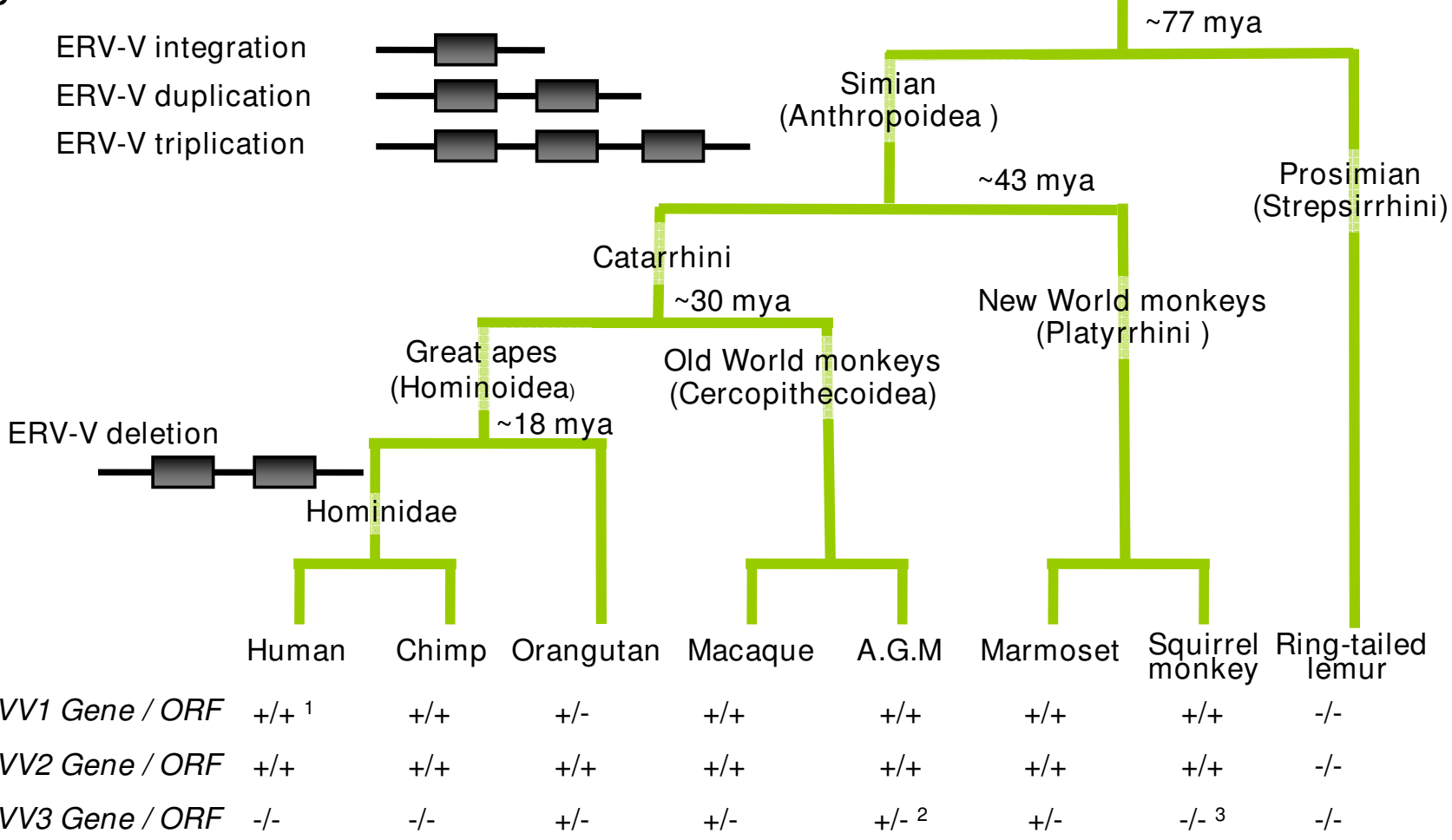

Figure 2

PCR identification of ENVV. A) ENVV2 PCR. Lane I: Human. Lane 2: B. orangutan. Lane 3: Africa green monkey. Lane 4: Squirrel monkey. Lane 5: Ring-tailed lemur. B) Primate phylogeny, with hypothetical integrations indicated. Below each species is indicated whether a given gene/ORF was detected (+) or not (-). Notes: I) Contains a 60 amino acid C-terminal truncation. 2) Detected by an internal ENVV PCR (see methods). 3) Not PCR detected.

conversion test [29] yielded highly significant results $(\mathrm{P}<$ 0.00066 , Table 1 ). The result indicates that gene conversion has taken place in all lineages, with 5 ' gene conversion breaks situated before the coding sequence or between position 1-45 as numbered from the initiator ATG, and 3' gene-conversion breaks between positions 1153-1312. To get an indication of the 5 ' gene conversion break, we extracted the variable sites from human, chimpanzee and macaque 5'UTRs. A shift in mutation pattern was observed around position -192, upstream of which the ERV-Vs follow the expected mutation pattern, whereas the pattern downstream of this position is consistent with gene conversion (data not shown).

When only looking at variable sites (Figure 3B) we note that the alignment is divided into two parts. The first part (Figure 3B, green) contains mutations that are shared by both paralogue and orthologue ENVV's within the Hominidae and Old World monkey lineage respectively, a pattern consistent with gene conversion. The resulting phylogenetic tree (Figure 3C, tree 1) shows ENVV sequences clustering into monophyletic groups, where ENVV1 and ENVV2 cluster together inside each group. This is consistent with this region of the ENVV2 locus being transferred to the ENVV1 locus (or vice versa) by gene conversion. The other part (Figure $3 \mathrm{~B}$, red) is consistent with a model of normal species-like evolution, which is supported by the corresponding phylogenetic tree (Figure 3C, tree 2) showing paralogue ENVV genes splitting into two groups.

\section{Evidence of purifying selection of the ENVV2 gene}

The ENVV2 envelope ORFs have been conserved since integration more than 40 mya (Figure $2 \mathrm{~B}$ ). In some spe- 
A

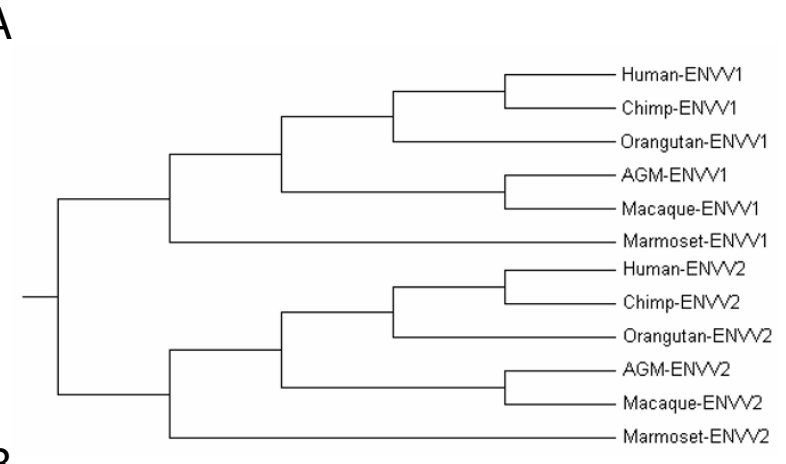

B

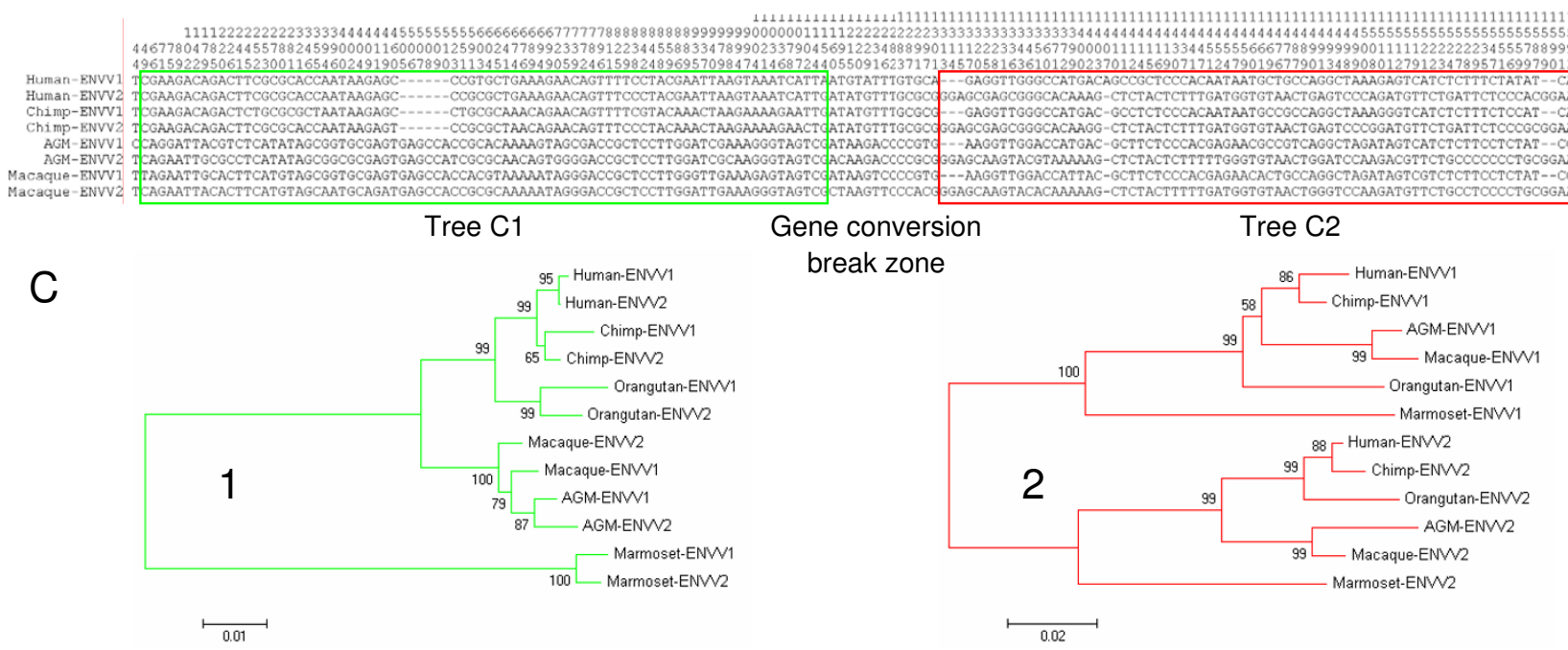

Figure 3

Gene conversion between the ENVVI and ENVV2 loci. Detection of gene conversion between the ENVVI and ENVV2 loci. A) Theoretical phylogenetic tree of normal species-like of evolution of ENVVI and ENVV2. B) Alignment of sequences from species containing conserved ENVVI and ENVV2, including variable sites only. A green box shows unexpected evolution and red box shows the expected evolution (following the phylogeny in $(A)$ ). The gene conversion break zone between the boxes is the region where 3' gene conversion breaks are predicted. C) Neighbor-joining phylogenetic tree (Maximum Composite Likelihood; 1000 Bootstrap) of the 45-II 32 region (green) and the I3 I2-end region (red).

Table I: Test of gene conversion between ENVVI and ENVV2

\begin{tabular}{lccccccc}
\hline \multirow{2}{*}{ ENVVI:ENVV2 } & \multicolumn{3}{c}{ Aligned Offsets } & & \\
P-value & Begin & End & Length & \# poly & \# dif & Total dif \\
\hline Human & 0.00000 & 1 & 1153 & 1153 & 195 & 2 & 64 \\
Chimpanzee & 0.00053 & 1 & 1312 & 1312 & 219 & 10 & 67 \\
Orangutan & 0.00003 & 5 & 1312 & 1308 & 218 & 19 & 80 \\
African gr. monkey & 0.00000 & 45 & 1296 & 1252 & 212 & 11 & 74 \\
Macaque & 0.00006 & 1 & 1290 & 1290 & 216 & 13 & 72 \\
Marmoset & 0.00066 & 1 & 1278 & 1278 & 210 & 12 & 69 \\
\hline
\end{tabular}

P-values are Bonferroni-adjusted Karlin-Altschul p-vaules. The positions are corresponding to the aligned positions in the ENVVI and ENVV2 sequences. "\# poly" is the number of polymorphic sites in the fragment. "\# dif" is the number of mismatches in the fragment. "Total dif" is the number of mismatch between ENVVI and ENVV2 in the species. 
cies both ERV loci contain an intact ENV gene (ENVV1 and ENVV2) whereas in other species the ENVV1 gene is degenerated.

All sequences show very high conservation within the ENVV2 region, whereas the similarity drops in flanking regions (Figure 4A). Additionally, the hydrophobicity profile and several characteristic motifs within gammaretroviruses are preserved in all full-length ENVV2s during evolution (Figure 4B). These include a CWIC motif involved in SU subunit and TM subunit interaction [30] and in controlling the fusion by a disulfide isomerization step [31], the cleavage site (RQKR) between SU and TM of the envelope gene, the hydrophobic domain in the N-terminus of TM corresponding to the fusion peptide, two heptad repeats in TM, involved in a conformational change in the envelope during fusion $[32,33]$, the CKS-17like immunosuppressive domain that has immunosup-

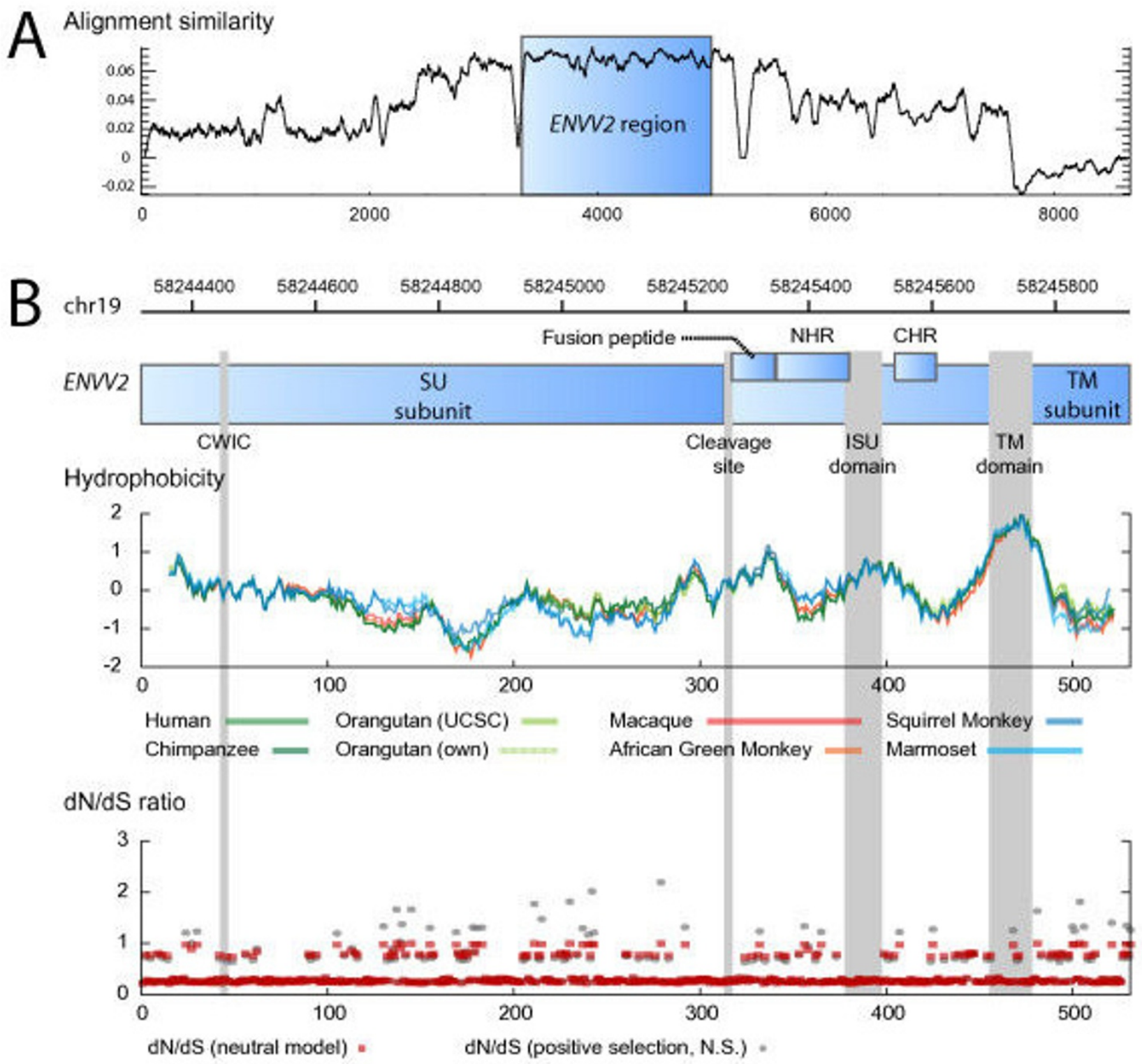

\section{Figure 4}

Purifying selection of ENVV2 sequences. (A) The ENVV2 reading frame (blue) is intact in all five primate genomes and shows a higher alignment similarity than the flanking regions. (B) Analysis of ENVV2 proteins, hydrophobicity plot for eight primate sequences. The most likely site specific $\mathrm{dN} / \mathrm{dS}$ ratios are shown for the accepted model incorporating two classes of $\mathrm{dN} /$ $\mathrm{dS}$ ratios (red) and the non-significant model of positive selection incorporating three classes (grey). 
pressive activity [11], and the transmembrane region anchoring the envelope protein to the membrane.

The HERV-V2 locus contains eight SINE elements all located closely outside ENVV2, both inside and between gag and pol fragments (Figure 5). Assuming a random distribution of SINE elements within the HERV-V2 locus (i.e. assuming no selection on the ENVV2 region), the probability of observing the 8 SINE elements outside and 0 SINE elements inside the ENVV2 gene is significant $(P=$ 0.048 ), indicating that the ENVV2 gene has survived transposon crossfire.

The entire envelope gene shows purifying selection between all pairs of species, but not between the two almost identical orangutan sequences when testing the ratio between nonsynonymous and synonymous substitutions ( $\mathrm{dN} / \mathrm{dS}$ ratio, Table 2). A site-specific selection analysis using PAML [34] provides strong support for a model with two site classes (M1 vs. $\mathrm{M0}: \chi^{2}=30.5, \mathrm{df}=2$, $p<1 \mathrm{e}-7$ ), one under strong purifying selection (mean $\mathrm{dN} /$ $\mathrm{dS}=0.075,63 \%$ of sites $)$ and one neutral class $(\mathrm{dN} / \mathrm{dS}=$ $1.000,37 \%$ of sites). Although some positions show signs of positive selection (Figure 4B), there is no significant support for a positive selection model (M2a vs. M1: $\chi^{2}=$ $1.46, \mathrm{df}=2, p=0.4819$ ).

Additionally, ENVV1 and ENVV2 have been shown to have a placenta-specific expression in humans [18] and our RT-PCR data confirmed this particular expression (data not shown). Interestingly, EST-data from both olive baboon (Papio anubis, Old World monkeys) and human verify the placental expression of ENVV1 and ENVV2 [35].

\section{Discussion}

The HERV-V1 and HERV-V2 loci display a remarkable evolution. They are the only two copies of the HERV-V family within the human genome, and there is no evidence of any solitary LTRs. However, the identified LTR regions are small and fragmented and there has been a long time span since integration. This prevents efficient recognition of solitary LTRs using BLAST, hence it is possible that they are present but remain undetected. In both HERV-V family members, the envelope genes (ENVV1 and ENVV2) consist of a long ORF. ERV-V1 and ERV-V2 are located in tandem in all species analyzed as a result of a gene duplication event. In all lineages except Hominidae, a third copy, ERV-V3, was detected between ERV-V1 and ERV-V2. The tandem location of this third copy is indicative of a gene triplication at the locus, however, this could not be directly proven, since no flanking sequence homologies were found.

The human ENVV1 shows a minor truncation at the C-terminus, due to a 1 nucleotide insert, leading to a frame shift, which has not been shown to be polymorphic in the human population, according to SNP databases (data not shown). However, the truncation has little impact on the structural prediction of the ENVV1 protein, nevertheless, the cytoplasmic tail is missing. Both ENVV1 and ENVV2 contain all structural motifs within envelope proteins reported to be important in viral-cell fusion. This suggests a similar function of the ENVV genes in the host by mediating cell-cell fusion, as has been observed for three other HERV envelopes (syncytin 1, syncytin 2 and EnvPb1 $[15,17,18])$. Phylogenetic studies show that the ERV-V family was fixed in an ancestral genome before the Catarrhini-Platyrrhini split $~ 43$ mya but after simians had evolutionarily separated from the prosimians ( 77 mya). Sequence analyses confirmed that ENVV2 is preserved among simians, and ENVV1 has been preserved in most species within Catarrhini, further all species except Hominidae enclose an extra ERV-V copy (ERV-V3), which seems to be lost in the Hominidae lineage (Figure 2B). A detailed comparative analysis of the ERV-V2 locus shows that the ENVV2 genes have been subjected to purifying selection (dN/dS $<1$, Table 3 and Figure 4B). Additionally, ENVV2 survival during transposon crossfire inside the ERV-V2 locus supports purifying selection of the ENVV2 gene. It is unlikely that the envelope gene has been maintained as a

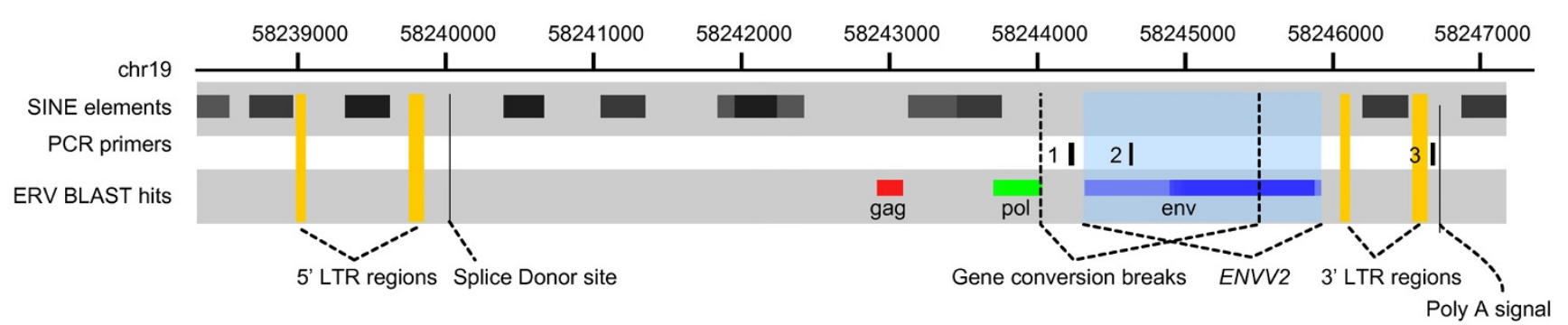

Figure 5

ENVV2 annotation. Annotation of the human HERV-V2 locus showing the high content of SINE elements in this region. Gag, pol and env tBLASTn hits are indicated by red, green and blue, predicted LTR regions (yellow). The region undergoing gene conversion is indicated by vertical dashed lines (see text). Splice donor site and poly A signal are predicted sites. 
Table 2: Codon-based Test of Purifying Selection between sequences

\begin{tabular}{lcccccccc}
\hline & H.s. & P.t. & P.p.a. & P.p. & M.m. & C.a. & S.s. & C.j. \\
\hline H.s. & & 2.456 & 2.707 & 2.661 & 3.244 & 4.466 & 3.967 & 5.856 \\
P.t. & 0.008 & & 3.852 & 3.750 & 3.665 & 4.862 & 4.290 & 5.939 \\
P.p.a. & 0.004 & 0.000 & & 0.978 & 3.044 & 4.253 & 4.362 & 6.381 \\
P.p. & 0.004 & 0.000 & 1.000 & & 2.986 & 4.143 & 4.167 & 6.225 \\
M.m. & 0.001 & 0.000 & 0.001 & 0.002 & & 4.289 & 3.706 & 6.282 \\
C.a. & 0.000 & 0.000 & 0.000 & 0.000 & 0.000 & & 4.927 & 7.217 \\
S.s. & 0.000 & 0.000 & 0.000 & 0.000 & 0.000 & 0.000 & & 6.005 \\
C.j. & 0.000 & 0.000 & 0.000 & 0.000 & 0.000 & 0.000 & 0.000 &
\end{tabular}

Test of purifying selection (codon-based Z-test). Upper triangle: Zvalue and the lower triangle $P$-values. Analyses were conducted using the Nei-Gojobori method and Z-value variance was computed using 500 bootstrap replicates. H.s.: Human (Homo sapiens); P.t.: Chimpanzee (Pan troglodytes); P.p.a.: S. orangutan (Pongo pygmaeus abelii); P.p.: B. orangutan (Pongo pygmaeus); M.m.: Macaque (Macaca mulatta); C.a.: African green monkey (Cercopithecus aethiops); S.s.: Squirrel monkey (Saimiri sciureus); C.j.: Marmoset (Callithrix jacchus)

result of retroviral replication through an extracellular cycle, since the sites of integration have been maintained through simian evolution. In conclusion, the results provide a strong argument of their beneficial role of the ENVV2 gene for the host.

ENVV1 and ENVV2 expression in placenta is particularly interesting since the two HERV envelope proteins (syncytin 1 and syncytin 2) having been shown to cause cell-cell fusion in vitro are also highly expressed in placenta $[15,17]$. Syncytin 1 appears to display an important role in placenta development [24] and syncytin 2 may have immunosuppressive properties in placenta [11]. Furthermore, EST data show placenta expression of ENVV1 and ENVV2 in olive baboon (Old World monkeys), which demonstrates that the placental expression is indeed conserved through simian evolution. The placenta-specific expression in combination with the purifying selection documented by our phylogenetic analysis indicates that ENVV2 (and/or ENVV1) is likely to play a physiological role in the placenta.

Comparative analysis of the two ERV-V loci suggests that gene conversion within the ENVV loci has taken place by DNA transfer of the major part of 5' region of ENVV2 to ENVV1 (or vice versa). Gene conversion seems to be a common event in this region, since it has taken place in all lineages (Table 1 and Figure 3 ). Gene conversion is a common event in evolution, and is favored between paralogue genes located on the same chromosome less than $55 \mathrm{~kb}$ apart [36] just as ENVV1 and ENVV2. Gene conversion has also been reported within other ERV families [27,37], however, none of them within coding viral genes. The gene conversion break points are located just upstream of the putative translation initiation site and downstream of the immunosuppressive domain (ISU) resulting in a $\sim 1,5$ $\mathrm{kb}$ gene conversion tract. Recent results have shown that the ISU of ENVV2 contains immunosuppressive activity [11], and we have shown that the ISU of ENVV1 and ENVV2 are preserved, indicating a conservation of immunosuppressive activity through evolution. Additionally, the study has shown that an envelope gene truncated after the ISU maintains the immunosuppressive activity [11]. In this manner, gene conversion in the Hominidae and Cercopithecidea lineage homogenizes the ENVV genes and induces two $E N V V$ genes, both having a structure compatible with preservation of immunosuppressive properties.

In most simians, ENVV1 and ENVV2 have been preserved, raising the possibility of a distinct biological function of their protein products which differ in their C-terminal parts. Such function is consistent with the finding that both loci are expressed in humans as well as in olive baboon. However, this hypothesis of a separate function of the two ENVV genes is not supported by phylogenetic analysis in simians as such, since orangutan and New World monkeys hold only one intact ENVV gene.

Neither ENVV2 or ENVV1 have been shown to be fusiogenic [18], but a fusiogenic property of $E N V V$ in placenta development cannot be excluded, since the selection pressure on the ENVV2 gene is not only restricted to the region necessary in having immunosuppressive activity, but includes the entire envelope gene. However, the fusiogenic properties may be activated by a physiological environment in placenta, like high-oxygen pressure has been found to regulate the pathway of cytotrophoblast differentiation $[38,39]$. Receptor recognition of the ENVV proteins may also be different to that of other envelope proteins such as syncytin 1, since the organization of the CWIC domain (involved in disulfide isomerisation during fusion [30,31]) (Figure 4B) within the SU is different. In $E N V V$ the CWIC motif is located in the $\mathrm{N}$-terminal part of the SU while it is located more C-terminally in syncytin 1 and envelope proteins of other gammaretroviruses. However this SU organization is not conflicting with envelope fusiogenic activity, since this organization is also observed in the fusiogenic syncytin 2 [10], syncytin A and syncytin B [40] ERV envelopes.

Another potential function of the ENVV genes could be the prevention of present-day infections by exogenous retroviruses through blocking the receptor used by the attacking virus, a process called receptor interference, as seen for other ERV envelope genes [13,14,41]. This possibility is consistent with the high sequence similarity and purifying selection of ENVV2 among species, but the hostreceptor and the receptor binding domain of the envelope protein are unknown. Notably, the gene conversion events have preserved two copies of the presumed ENVV receptor binding domain, located in the $\mathrm{N}$-terminal part 
of SU in all known retroviruses. Moreover, in all species encoding an ENVV1 protein this is predicted to be membrane-anchored and may thus potentially have receptorinterference activity. This is also the case for humans where the C-terminal truncation ENVV1 does not abrogate membrane anchoring.

\section{Conclusion}

Our analyses have shown that the ERV-V family is small, consisting of two copies in Hominidae. However, orangutan, Old World monkeys and New World monkeys include an extra copy, all located in tandem on chromosome 19. The ERV-V2 envelope ENVV2 has been preserved for more than 43 million years in all species tested. Within Hominidae and Old World monkeys the ENVV1 has been preserved by gene conversion of a $1.5 \mathrm{~kb}$ region encoding the N-terminal part of ENVV2, meaning that they contain two copies of the ENVV envelope, both expressed in placenta. These data provide strong evidence of a beneficial role for the host in placenta. A likely function of the ENVV gene could be: i) Protecting the fetus against the immune system of the mother, due to immunosuppressive properties [11]. ii) Mediating cell fusion and making of the syncytiotrophoblast. iii) Blocking a retroviral receptor through receptor-interference and thereby preventing present-day retroviral infections.

\section{Methods}

\section{DNA samples, PCR amplification, and sequencing}

DNA from African green monkey (Cercopithecus aethiops), Borneo orangutan (Pongo pygmaeus) and squirrel monkey (Saimiri sciureus) was isolated from cell cultures (using Invitrogen DNAzol), tissue from ring-tailed lemur (Lemur catta) was a gift from Living United/Ebeltoft Zoo \& Safari (DNA was isolated using a QIAGEN tissue isolation kit). All primate DNA samples were verified by amplification and direct sequence analysis of the cytochrome oxidase I (COI) gene. Sequences from human (Homo sapiens), chimpanzee (Pan troglodytes), rhesus monkey (Macaca mulatta), Sumatran orangutan (Pongo pygmaeus abelii) were extracted from the ENSEMBL genome database http://www.ensembl.org[42], marmoset sequences were extracted from the UCSC Genome Browser Primers, (1) "ENVV1/V2 forward" CAGCCTGATTTCTCACTAAACACT CCATCGAAC, "ENVV1 reverse" CTCAGCGTGCAGCGTTTCCAAGGAGGCCATCAGCG, (3) "ENVV2 reverse" CTAG TGCCTTAGTTTTTATGGGAGCT, were designed to amplify a $\sim 2.5 \mathrm{~kb}$ fragment, on both sides of either ENVV1 or ENVV2. The PCR was performed by the use of expand high fidelity PCR system (Roche) in $100 \mu \mathrm{l}$ reaction using $300 \mathrm{nM}$ primer, $0.2 \mathrm{mM}$ dNTP, $150 \mathrm{ng}$ of DNA, and buffers by the supplier. The PCR program was as follows: 1 cycle $\left(94^{\circ} \mathrm{C}\right.$ for $\left.1 \mathrm{~min}\right), 30$ cycles $\left(94^{\circ} \mathrm{C}\right.$ for $1 \mathrm{~min}$; $60^{\circ} \mathrm{C}$ for $1 \mathrm{~min} ; 72^{\circ} \mathrm{C}$ for $\left.2.5 \mathrm{~min}\right)$, and 1 cycle $\left(72^{\circ} \mathrm{C}\right.$ for $10 \mathrm{~min})$. The internal ENVV PCR was done by the same procedure by using primer (1) and the "ENVV internal" primer (2) CTAACATTTGGTTCAGGAATCC. Resulting PCR products were either directly sequenced from a $5-\mu \mathrm{l}$ PCR mixture or after cloning of the fragment into pGEMTeasy (Promega) using $2 \mu \mathrm{l}$ of PCR product. Sequencing were performed using the BigDye ${ }^{\circledast}$ Terminator v3.1 Cycle Sequencing Kit (Applied Biosystems) (details for sequence primers are available from the authors upon request) and analyzed on an ABI3100 (Applied Biosystems). All conserved ENVV1 and ENVV2 ORFs have been deposited in GenBank under the accession numbers EU853142 and EU853155.

\section{LTR analysis}

We tried dating the integration using the most distant species (human and marmoset) to minimize the effect of uncertainty in known speciation times. LTR regions were identified in the ERV-V2 locus in human and marmoset using dot plots, Repeatmasker data, and genomic BLAT searches. The alignment was cropped to only include sites with high alignment quality resulting in only $155-158 \mathrm{bp}$ that aligned unambiguously between species. LTR divergence was calculated in MEGA4 [43] using Tamura 3parameter distance allowing different rates among lineages (since it is easily observed that 3 ' LTRs are a lot more divergent than 5' LTRs between species) and different rates among sites (gamma $=1.0)$. The integration time is estimated as:

$$
\text { Time }_{\text {integration }}=\frac{\text { divergence }_{5^{\prime}-3^{\prime} \text { LTR }}}{\text { rate }_{5^{\prime} \mathrm{LTR}^{+} \text {rate }_{3} \mathrm{~L}^{\prime} \mathrm{LTR}} .}
$$

We used species-pairs to estimate integration time from independent 5' and 3' LTR substitution rate estimates. E.g. for the human-chimp pair, we estimated the H-C 5' LTR substitution rate from the observed H-C 5' LTR divergence and published $\mathrm{H}-\mathrm{C}$ speciation time ( $\sim 5 \mathrm{mya}$ ). The human-macaque pair yields a slightly different estimate and so forth.

Sequences were aligned using ClustalW [44] and GENECONV [29] was used to predict gene conversion. We extracted variable sites using FaBox [45]. Phylogenies (Neighbor-joining phylogenetic tree, Model: Maximum Composite Likelihood; 1000 Bootstrap replicates) and evolutionary distances were calculated using MEGA4 [43].

The ENVV2 DNA alignment similarity plot was created using the EMBOSS plotcon program [46] with a sliding window of size 32 and EDNAFULL as similarity matrix (figure 4A). The protein hydrophobicity plots was created using EMBOSS pepwindowall program [46] with a sliding window of size 30 (figure 4B). SINE elements in the human ERV-V2 locus were identified using Repeatmasker [47] and ENVV2 selection tests were performed using 
MEGA4 [43] (Nei-Gojobori method and Z-value variance was computed using the 500 bootstrap replicates). PAML4 [34] was used to run site-specific selection tests and obtain $\mathrm{dN} / \mathrm{dS}$ ratios from all ENVV2 sequences. The models analyzed assumed no molecular clock ( clock $=0)$, a single omega for all tree branches (model $=0$ ) and we used likelihood ratio tests to compare the improvement in likelihood for models incorporating 1, 2 or 3 site classes (NSsites = $\left.\begin{array}{lll}0 & 1 & 2\end{array}\right)$. Each analysis ran until convergence (Small_Diff $=0.5 e-6$ ) and the control file is available upon request.

\section{Abbreviations}

ERV: Endogenous retrovirus; EST: Expressed Sequence Tags; HERV: Human endogenous retrovirus; ISU: Immunosuppressive domain; LTR: Long terminal repeats; mya: Million years ago; PCR: Polymerase chain reaction; SNP: Single-nucleotide polymorphism; SU: Surface glycoprotein; TM: Transmembrane protein

\section{Authors' contributions}

ALK drafted the manuscript, performed all experimental work and performed the statistical and evolutionary analysis. PV participated in sequence manipulations, statistical and evolutionary analyses, prepared figures, and drafted the manuscript. LAA and FSP coordinated the study and participated in critical revision of the manuscript. All authors read and approved the final manuscript.

\section{Acknowledgements}

This work was supported by two grants from the Lundbeck Foundation (PV and FSP). The authors thank Jesper Stagegaard (Living United/Ree Park) for providing primate DNA samples.

\section{References}

I. Sverdlov ED: Perpetually mobile footprints of ancient infections in human genome. FEBS Lett 1998, 428: I-6.

2. Belshaw R, Pereira V, Katzourakis A, Talbot G, Paces J, Burt A, Tristem M: Long-term reinfection of the human genome by retroviruses endogenous. Proc Natl Acad Sci USA 2004, | 0 | :4894-4899.

3. Löwer R, Löwer J, Kurth R: The viruses in all of us: characteristics and biological significance of human endogenous retrovirus sequences. Proc Natl Acad Sci USA 1996, 93:5।77-5। 84

4. Boeke JD, Stoye JP: Retrotransposons, endogenous retroviruses, and the evolution of retroelements. In Retroviruses Edited by: Coffin JM, Hughes SH, Varmus HE. Cold Spring Harbor Press, NY; 1997:343-435.

5. Shih A, Coutavas EE, Rush MG: Evolutionary implications of primate endogenous retroviruses. Virology 1991, I 82:495-502.

6. Steiper ME, Young NM: Primate molecular divergence dates. Mol Phylogenet Evol 2006, 41:384-394.

7. Turner G, Barbulescu M, Su M, Jensen-Seaman MI, Kidd KK, Lenz J: Insertional polymorphisms of full-length endogenous retroviruses in humans. Curr Biol 2001, I I:I53 I-I535.

8. Dewannieux M, Harper F, Richaud A, Letzelter C, Ribet D, Pierron G, Heidmann $T$ : Identification of an infectious progenitor for the multiple-copy herv-k human endogenous retroelements. Genome Res 2006, I6:1548-1556.

9. Lee YN, Bieniasz PD: Reconstitution of an infectious human endogenous retrovirus. PLoS Pathog 2007, 3:el0.
10. de Parseval N, Lazar V, Casella J, Benit L, Heidmann T: Survey of human genes of retroviral origin: identification and transcriptome of the genes with coding capacity for complete envelope proteins. J Virol 2003, 77:104|4-10422

II. Mangeney M, Renard M, Schlecht-Louf G, Bouallaga I, Heidmann O, Letzelter C, Richaud A, Ducos B, Heidmann T: Placental syncytins: genetic disjunction between the fusogenic and immunosuppressive activity of retroviral envelope proteins. Proc Natl Acad Sci USA 2007, 104:20534-20539.

12. Mangeney M, de Parseval N, Thomas G, Heidmann T: The fulllength envelope of an herv-h human endogenous retrovirus has immunosuppressive properties. I Gen Virol 200I, 82:25I5-25|8.

13. Ponferrada VG, Mauck BS, Wooley DP: The envelope glycoprotein of human endogenous retrovirus herv-w induces cellular resistance to spleen necrosis virus. Arch Virol 2003, I 48:659-675.

14. Best S, Le Tissier PR, Stoye JP: Endogenous retroviruses and the evolution of resistance to retroviral infection. Trends Microbiol 1997, 5:313-318.

15. Mi S, Lee X, Li X, Veldman GM, Finnerty H, Racie L, LaVallie E, Tang $X Y$, Edouard P, Howes S, Keith JCJ, McCoy JM: Syncytin is a captive retroviral envelope protein involved in human placental morphogenesis. Nature 2000, 403:785-789.

16. Blond JL, Lavillette D, Cheynet V, Bouton O, Oriol G, Chapel-Fernandes S, Mandrand B, Mallet F, Cosset FL: An envelope glycoprotein of the human endogenous retrovirus herv-w is expressed in the human placenta and fuses cells expressing the type d mammalian retrovirus receptor. J Virol 2000 74:332I-3329.

17. Blaise S, de Parseval N, Bénit L, Heidmann T: Genomewide screening for fusogenic human endogenous retrovirus envelopes identifies syncytin 2 , a gene conserved on primate evolution. Proc Natl Acad Sci USA 2003, 100:13013-13018.

18. Blaise S, de Parseval N, Heidmann T: Functional characterization of two newly identified human endogenous retrovirus coding envelope genes. Retrovirology 2005, 2:19.

19. Bonnaud B, Bouton O, Oriol G, Cheynet V, Duret L, Mallet F: Evidence of selection on the domesticated ervwe $I$ env retroviral element involved in placentation. Mol Biol Evol 2004, 21:1895-1901.

20. Aagaard L, Villesen P, Kjeldbjerg AL, Pedersen FS: The approximately 30-million-year-old ervpbI envelope gene is evolutionarily conserved among hominoids and old world monkeys. Genomics 2005, 86:685-691.

21. de Parseval N, Diop G, Blaise S, Helle F, Vasilescu A, Matsuda F, Heidmann T: Comprehensive search for intra- and inter-specific sequence polymorphisms among coding envelope genes of retroviral origin found in the human genome: genes and pseudogenes. BMC Genomics 2005, 6:1 I7.

22. Malassiné A, Blaise $S$, Handschuh $K$, Lalucque $H$, Dupressoir A, EvainBrion D, Heidmann T: Expression of the fusogenic herv-frd env glycoprotein (syncytin 2) in human placenta is restricted to villous cytotrophoblastic cells. Placenta 2007, 28: 185-19|.

23. Malassiné A, Handschuh K, Tsatsaris V, Gerbaud P, Cheynet V, Oriol G, Mallet F, Evain-Brion D: Expression of herv-w env glycoprotein (syncytin) in the extravillous trophoblast of first trimester human placenta. Placenta 2005, 26:556-562.

24. Frendo J, Olivier D, Cheynet V, Blond J, Bouton O, Vidaud M, Rabreau $M$, Evain-Brion D, Mallet F: Direct involvement of herv-w env glycoprotein in human trophoblast cell fusion and differentiation. Mol Cell Biol 2003, 23:3566-3574.

25. Villesen P, Aagaard L, Wiuf C, Pedersen FS: Identification of endogenous retroviral reading frames in the human genome. Retrovirology 2004, I:32.

26. Hughes JF, Coffin JM: Human endogenous retroviral elements as indicators of ectopic recombination events in the primate genome. Genetics 2005, I7 I: I I83-1 I94.

27. Johnson WE, Coffin JM: Constructing primate phylogenies from ancient retrovirus sequences. Proc Natl Acad Sci USA 1999. 96: 10254-10260.

28. Blaise S, Ruggieri A, Dewannieux M, Cosset F, Heidmann T: Identification of an envelope protein from the frd family of human endogenous retroviruses (herv-frd) conferring infectivity and functional conservation among simians. J Virol 2004, 78: $1050-1054$ 
29. Sawyer S: Statistical tests for detecting gene conversion. Mol Biol Evol 1989, 6:526-538.

30. Pinter A, Kopelman R, Li Z, Kayman SC, Sanders DA: Localization of the labile disulfide bond between su and tm of the murine leukemia virus envelope protein complex to a highly conserved cwlc motif in su that resembles the active-site sequence of thiol-disulfide exchange enzymes. J Virol 1997, 71:8073-8077.

31. Wallin M, Ekström M, Garoff H: Isomerization of the intersubunit disulphide-bond in env controls retrovirus fusion. $E M B O J$ 2004, 23:54-65.

32. Melikyan GB, Markosyan RM, Hemmati H, Delmedico MK, Lambert DM, Cohen FS: Evidence that the transition of hiv-I gp4I into a six-helix bundle, not the bundle configuration, induces membrane fusion. J Cell Biol 2000, I 5 I:4 |3-423.

33. Renard M, Varela PF, Letzelter C, Duquerroy S, Rey FA, Heidmann T: Crystal structure of a pivotal domain of human syncytin-2, a 40 million years old endogenous retrovirus fusogenic envelope gene captured by primates. J Mol Biol 2005, 352: I029-1034.

34. Yang Z: Paml 4: phylogenetic analysis by maximum likelihood. Mol Biol Evol 2007, 8:|586-159|.

35. UCSC genome browser [http://genome.ucsc.edu/]

36. Ezawa $K$, OOta $S$, Saitou N: Proceedings of the smbe trinational young investigators' workshop 2005. genome-wide search of gene conversions in duplicated genes of mouse and rat. Mol Biol Evol 2006, 23:927-940.

37. Bosch E, Hurles ME, Navarro A, Jobling MA: Dynamics of a human interparalog gene conversion hotspot. Genome Res 2004, 1 4:835-844.

38. Robins JC, Heizer A, Hardiman A, Hubert M, Handwerger S: Oxygen tension directs the differentiation pathway of human cytotrophoblast cells. Placenta 2007, 28: I|4|-I| 46.

39. Kudo Y, Boyd CAR, Sargent IL, Redman CWG: Hypoxia alters expression and function of syncytin and its receptor during trophoblast cell fusion of human placental bewo cells: implications for impaired trophoblast syncytialisation in preeclampsia. Biochim Biophys Acta 2003, 1638:63-7I.

40. Dupressoir A, Marceau G, Vernochet C, Bénit L, Kanellopoulos C, Sapin V, Heidmann T: Syncytin-a and syncytin-b, two fusogenic placenta-specific murine envelope genes of retroviral origin conserved in muridae. Proc Natl Acad Sci USA 2005, 102:725-730.

4I. Gardner MB, Kozak CA, O'Brien S): The lake casitas wild mouse: evolving genetic resistance to retroviral disease. Trends Genet 1991, 7:22-27.

42. Ensembl genome browser [http://www.ensembl.org/]

43. Tamura K, Dudley J, Nei M, Kumar S: Mega4: molecular evolutionary genetics analysis (mega) software version 4.0. Mol Biol Evol 2007, 24: 1596-1599.

44. Thompson JD, Higgins DG, Gibson TJ: Clustal w: improving the sensitivity of progressive multiple sequence alignment through sequence weighting, position-specific gap penalties and weight matrix choice. Nucleic Acids Res 1994, 22:4673-4680.

45. Villesen P: Fabox: an online toolbox for fasta sequences. Molecular Ecology Notes 2007, 7:965-968.

46. Rice P, Longden I, Bleasby A: Emboss: the european molecular biology open software suite. Trends Genet 2000, 16:276-277.

47. Repeatmasker web server [http://www.repeatmasker.org/]
Publish with BioMed Central and every scientist can read your work free of charge

"BioMed Central will be the most significant development for disseminating the results of biomedical research in our lifetime. "

Sir Paul Nurse, Cancer Research UK

Your research papers will be:

- available free of charge to the entire biomedical community

- peer reviewed and published immediately upon acceptance

- cited in PubMed and archived on PubMed Central

- yours - you keep the copyright

Submit your manuscript here:

http://www.biomedcentral.com/info/publishing_adv.asp
BioMedcentral 